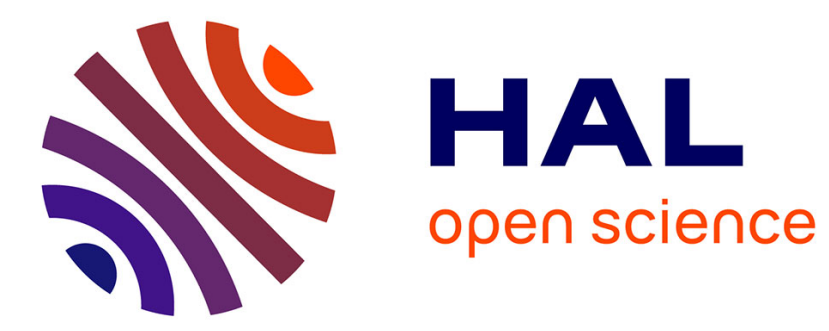

\title{
Réfugié, immigré, minorité: des mots pour penser les relations interethniques
}

Hélène Bertheleu

\section{To cite this version:}

Hélène Bertheleu. Réfugié, immigré, minorité: des mots pour penser les relations interethniques. Hommes \& migrations, 2001, 1234 (1), pp.23-32. 10.3406/homig.2001.4827 . halshs-02614623

\section{HAL Id: halshs-02614623 \\ https://shs.hal.science/halshs-02614623}

Submitted on 21 May 2020

HAL is a multi-disciplinary open access archive for the deposit and dissemination of scientific research documents, whether they are published or not. The documents may come from teaching and research institutions in France or abroad, or from public or private research centers.
L'archive ouverte pluridisciplinaire HAL, est destinée au dépôt et à la diffusion de documents scientifiques de niveau recherche, publiés ou non, émanant des établissements d'enseignement et de recherche français ou étrangers, des laboratoires publics ou privés. 


\title{
Réfugié, immigré, minorité : des mots pour penser les relations interethniques
}

\author{
Hélène Bertheleu \\ sociologue \\ Ville Société Territoire, \\ Université de Tours
}

H. Bertheleu - 2001 - "Réfugié, immigré, minorité : des mots pour penser les relations interethniques", in France, Terre d'Asie, Hommes et Migrations, n²1234, nov.-déc. 2001, p. 23 32.

Juridiquement comme émotionnellement, une distinction notable est établie, en France, entre "réfugiés" et "immigrés", y compris par les intéressés eux-mêmes. Les uns comme les autres constituent des groupes minoritaires confrontés à la norme majoritaire, confrontation qui influence aussi les processus d'identification desdites "minorités". À travers notamment l'exemple des réfugiés lao à Rennes, l'auteur montre ces processus et ces perceptions de la différence.

Afin de comprendre les conditions de vie et les relations sociales que les réfugiés d'Asie du SudEst ont construites depuis leur arrivée en France, il est prudent de revenir sur les termes communément utilisés que sont "réfugié", "immigré", "minorité". En sociologie, on est sans cesse confronté à la difficulté de nommer l'Autre sans l'essentialiser, au souci d'échapper à l'ethnocentrisme naiff qui accompagne tout processus d'altérisation, et au souci de l'analyser. Ces différents termes renvoient en effet à des catégories socialement et historiquement construites, elles font l'objet de définitions et de redéfinitions, souvent de débats. Quels types de rapports sociaux et quelles représentations de ces rapports aperçoit-on derrière ces termes ? Quelles images, quelles idées y sont véhiculées et que cela nous apprend-il sur les populations ainsi désignées ?

La figure de l'Étranger, qui s'est juridiquement construite et figée avec l'affirmation de l'Étatnation, se dédouble en France, selon la cause présumée de la migration, en deux catégories : réfugié et immigré. On utilise généralement le terme "réfugié" dans son sens juridique tel qu'il a été internationalement défini. Est réfugié celui ou celle qui a obtenu, après l'avoir sollicité, le statut de réfugié politique. C'est un statut international unique, général, qui implique protection et assistance de la part de l'État d'accueil. Il fait suite à la très ancienne tradition de l'asile, qui renvoyait à des considérations souvent religieuses, politiques ou philosophiques, et non à une question de droit (Convention de Genève, 1951).

Après la Seconde Guerre mondiale, ce système international de protection fut élaboré en référence aux événements qui venaient de se dérouler : on pensait qu'il s'agissait de régler les suites du conflit mondial et qu'ensuite les sources d'exil se tariraient. La Convention de Genève apparut après coup comme "européocentrée", c'est-à-dire que l'on imaginait avoir affaire à des individus qui, seuls ou avec quelques autres personnes, étaient contraints de quitter leur pays pour fuir les persécutions. Cet optimisme fut rapidement démenti par les faits car les flux de réfugiés furent beaucoup plus importants et diversifiés qu'on ne l'avait anticipé. Le problème des réfugiés est aujourd'hui considéré comme une donnée permanente de la vie internationale et non plus un phénomène lié à la situation exceptionnelle que fut la Seconde Guerre mondiale.

Suite aux événements qui touchèrent les trois pays de l'ex-Indochine au cours des années soixante-dix, les réfugiés quittant le Cambodge, le Laos et le Vietnam furent nombreux et il fallut 
imaginer une solution globale à la question. Au niveau des États d'abord, contraints de collaborer pour mener une action collective d'assistance aux réfugiés, puis en France, pour mettre en place des structures d'accueil de ces populations et leur permettre d'obtenir rapidement le statut de réfugié, par le biais de mesures spéciales de traitement collectif.

On connaît les conditions qui permettent d'obtenir, en principe, le statut de réfugié :

$1^{\circ}$ Avoir franchi une frontière ;

$2^{\circ}$ Avoir été persécuté ou avoir des raisons fondées de croire qu'on le sera ;

$3^{\circ}$ Que ces persécutions portent atteinte aux droits fondamentaux de cette personne ;

$4^{\circ} \mathrm{Qu}$ 'elle ne puisse, de ce fait, se réclamer de la protection de l'État dont elle porte la nationalité et/ou où elle réside ordinairement.

\section{Opposition sémantique entre "réfugié" et "immigré"}

En France, c'est l'Office français pour les réfugiés et les apatrides (Ofpra) qui étudie les dossiers de demande d'asile et octroie le statut de réfugié, en fonction d'une décision sur le caractère fondé ou non de la crainte de persécutions. On sait que le problème n'est pas si simple, que la définition juridique du réfugié est restrictive et que les réfugiés qui obtiennent le statut ne sont pas les seuls à avoir besoin d'asile, de protection et d'assistance. On emploie communément mais abusivement l'expression "droit d'asile" : il serait plus correct, étant donné la situation réelle faite aux demandeurs d'asile, de parler de "droit à rechercher l'asile" (ce que garantit en principe le droit au non-refoulement à la frontière) pour ne pas laisser croire qu'il s'agit d'un droit à l'asile sans condition.

Il est intéressant de se pencher, à ce propos, sur des travaux récents comme ceux d'Olivier Brachet(1), qui montrent que les procédures auxquelles doit recourir quelqu'un pour se réfugier en France sont de plus en plus nombreuses et opaques. Le requérant est en effet obligé d'emprunter des circuits administratifs complexes, difficiles à comprendre. L'auteur observe d'autre part le développement de situations "marginales", d'une sorte d' "asile au noir", la multiplication de situations où les personnes ne sont pas officiellement admises sur le territoire mais y sont, de fait, tolérées. Selon son analyse, cet "asile au noir" est l'effet connu et délibéré d'une politique ; il est d'autant mieux toléré qu'il concerne, en France, un nombre de personnes beaucoup plus restreint que dans les autres pays d'Europe. Il est interprété alors comme une réponse "pragmatique" de la France s'inscrivant dans une politique de dissuasion migratoire qui vise à rendre très précaires les situations de ceux dont on pense qu'ils se lasseront et transmettront autour d'eux le message des difficultés rencontrées. La gestion de l'asile en France apparaît ici en partie régalienne et discrétionnaire.

Quand on est attentif aux divers discours et aux représentations sociales relatives à ces questions, on est frappé par l'opposition sémantique qui lie, en France, les termes "réfugié" et "immigré", opposition largement partagée, y compris par les personnes concernées. Au cours de mes observations et entretiens menés auprès des réfugiés lao(2), il m'a été donné plusieurs fois de constater l'importance de cette opposition dans la perception de soi et les réticences de mes interlocuteurs devant les situations où les deux "figures" risquaient d'être confondues. Ce fut le cas, par exemple, lorsque l'Association lao de Rennes fut sollicitée pour participer à des activités de quartier du type "échanges de savoirs", ou lors de la fête annuelle de la "diversité culturelle" qui, justement, s'appelait naguère "fête de l'immigration". Perçues comme autant de communautés culturelles riches en couleurs et en particularités, les diverses collectivités ethniques sont en effet invitées chaque année à cette manifestation, où chaque "culture" tient un stand, propose ses spécialités culinaires et artistiques. Les Lao ont participé une seule fois à cette manifestation et s'y sont sentis mal à l'aise : ils y ont vu une volonté explicite d'amalgamer leur situation à celle, peu valorisante à leurs yeux, des immigrés, tout en étant considérés, du point de vue des organisateurs, également "différents" et "particuliers" : la différence culturelle, on en prend la mesure ici, est inégalement connotée. Ils se sont sentis prisonniers d'un programme et 
d'une présentation de soi dont ils n'étaient pas familiers et ont préféré, par la suite, fréquenter les Français à l'intérieur de leurs propres animations et manifestations.

\section{La présence plus légitime des réfugiés}

En effet, les réfugiés lao, dont beaucoup ont opté aujourd'hui pour la nationalité française, préfèrent les situations par lesquelles ils maîtrisent davantage l'image qu'ils souhaitent donner d'eux-mêmes et de leur collectivité. Lors des grandes fêtes organisées à l'occasion du Nouvel An lao, les leaders associatifs n'hésitent pas, par exemple, à appeler un journaliste du quotidien Ouest-France pour qu'il informe la population rennaise de cette fête importante de leur calendrier, et qu'il diffuse à l'occasion un portrait valorisant de leur "communauté". Le journal participe ainsi au travail de sensibilisation de la population rennaise sur la situation des réfugiés lao en France. Il n'est pas rare que l'on trouve dans ces encarts une allusion à l'existence pacifique et discrète des réfugiés d'Asie du Sud-Est à Rennes, suggérée par le porte-parole interviewé ou bien rédigée par le journaliste lui-même, qui cède alors à une description certes simpliste, mais qui se veut surtout bienveillante. La comparaison avec les "immigrés" et les trop fameux "issus de l'immigration" est sous-jacente, mais elle est tue.

C'est la cause présumée de la migration que l'on souligne généralement pour distinguer les réfugiés des immigrés : fuite involontaire et forcée d'un côté, décision volontaire de migrer de l'autre. Migration volontaire ou involontaire, facteurs économiques ou politiques déterminant la migration, la "motivation" du migrant est retenue pour établir la distinction. Pourtant, les sociologues savent combien ce critère de la volonté de migrer est incertain. La plupart des migrations sont des réponses à des situations complexes où se mêlent des facteurs politiques, sociaux, économiques, mais aussi souvent ethniques ou religieux. Seuls les étudiants et les professionnels qualifiés échappent sans doute à ce constat.

Aux yeux de la population française, la présence du réfugié apparaît d'emblée plus légitime que celle de l'immigré ou même de l'enfant d'immigré, et ceci est particulièrement vrai pour les réfugiés d'Asie du Sud-Est. Il y eut une forte mobilisation pour les accueillir à leur arrivée, qu'il faut imputer en partie au sentiment de culpabilité de la France vis-à-vis de ses anciennes colonies, où son influence était encore forte peu d'années avant les événements de 1975. En 1979, la campagne médiatique de sensibilisation qui se déploya eut pour effet, en Ille-et-Vilaine, de susciter un véritable élan de solidarité vis-à-vis de ces réfugiés perçus comme les victimes par excellence de la guerre et du communisme. Entre 1979 et 1981, soixante-cinq groupes se constituèrent dans le département, permettant l'accueil de quatre-vingt-onze familles(3). Dix, quinze, voire vingt ans plus tard, les réfugiés se souviennent encore très bien de ces personnes qui ont contribué à la formation, dans leur esprit, d'une nouvelle idée des Français. Les relations entre les "parrains" et les familles accueillies ont été abondamment décrites : teintées d'abord de pitié puis de paternalisme, elles furent souvent ambiguës, parfois conflictuelles lorsque les comportements ou les décisions des chefs de famille (en matière de budget familial, de scolarité des enfants ou de choix professionnel) n'allaient pas dans le sens de "l'intégration" telle qu'elle avait été anticipée par les tenants de l'accueil. Plusieurs années après, certains membres de ces groupes d'accueil ont gardé des relations épisodiques avec "leur" famille ou avec l'association.

\section{Des représentations élaborées de la communauté}

Dès l'arrivée des réfugiés, les "anciens d'Indochine" aussi se sont manifestés, soit en contribuant directement à la mise en place et au fonctionnement des centres provisoires d'hébergement $(\mathrm{CPH})$, soit en participant aux groupes d'accueil et autres actions de soutien, puis, plus tard, proposant aux réfugiés de tisser un réseau de relations favorisant l'entraide en France et l'aide humanitaire dans l'ex-Indochine. Ce regard bienveillant (même s'il est aussi parfois pesant) n'est pas sans conséquence sur la manière dont se déroulent et se vivent les relations interethniques : le pouvoir de catégorisation et d'assignation identitaire dont dispose le groupe majoritaire tend à définir, pour l'étranger lui-même, le statut de sa migration. Pourrait-on ainsi contribuer à 
expliquer qu'à Rennes par exemple, les réfugiés se mettent plus volontiers en représentation devant les Français que ne le font les immigrés ?

Depuis le début des années quatre-vingt, les Lao organisent régulièrement de grandes fêtes où, parmi de nombreuses autres relations amicales et professionnelles, ces anciens militaires sont invités. C'est l'occasion pour l'association de proposer une représentation élaborée de la "communauté laotienne" de Rennes : cette image est travaillée, discutée collectivement, donne lieu à des débats sur ce qu'il est bon de montrer publiquement ou non, sur l'identité collective, finalement, que l'association souhaite promouvoir. Les choses ne sont pas dites aussi explicitement par les acteurs associatifs mais divers comportements (relatifs au jeu ou aux manières de boire, par exemple), diverses pratiques (culinaires, corporelles, musicales) et tenues vestimentaires ont été collectivement passées en revue pour décider si oui ou non elles avaient leur place au sein de ces grandes représentations, leur pertinence identitaire étant évaluée à travers une grille où le regard de l'Autre, c'est-à-dire le groupe majoritaire, était omniprésent. Les uns défendent une posture de retrait, une certaine discrétion ("il ne faut pas trop se montrer"), pensant ainsi déjouer la xénophobie latente, tandis que d'autres ont à cœur de faire découvrir aux Rennais leur culture originale, leurs valeurs (sélectionnées, il est vrai, pour leur succès potentiel), leur folklore, mais aussi d'engager des relations de qualité, symbole à leurs yeux d'une véritable insertion locale, rassurantes pour l'avenir. L'un d'eux dit les choses ainsi: “Je souhaite seulement que les gens nous connaissent, nous comprennent, et nous soutiennent en cas de pépin."

On peut aussi mesurer l'attitude de la population française à l'égard des réfugiés à travers l'approche statistique qui en fut faite. Dans une enquête réalisée en 1985(4), soit dix ans après les premières arrivées de réfugiés d'Asie du Sud-Est à Rennes, l'échantillon représentatif de la population rennaise s'exprime plutôt favorablement à l'égard des "Asiatiques" qui, dans les discours, apparaissent fréquemment en comparaison ou en opposition avec les "Maghrébins". La moitié des enquêtés pensent par exemple que pour les "Asiatiques", s'adapter en France est facile, tandis que seulement $8 \%$ font la même estimation pour les "Maghrébins".

\section{Un statut qui n'est pas immuable}

Enfin, un dernier exemple permettra de saisir la nature du regard porté sur ces populations alors qu'elles étaient récemment arrivées, comparé aux réactions que suscitent les initiatives des populations marocaines de Rennes. Au début des années quatre-vingt, la municipalité de Rennes s'est montrée favorable à la construction d'un centre culturel islamique à l'usage des musulmans de la ville. Cette décision déclencha une flambée de violence verbale de la part de quelques personnes extrémistes qui cherchaient à faire échouer le projet. L'enquête de 1985 précitée reflète non pas l'animosité à l'égard du projet mais bien l'ampleur de la polémique : tout le monde a entendu parler de la mosquée. Les opinions sont toutefois nuancées : on y trouve des réticences à voir les fonds publics contribuer à financer un lieu de culte et des craintes quant à l'idée d'encourager peut-être ainsi une vie trop communautaire ; mais une majorité de répondants reste plutôt favorable à ce geste d'ouverture et de reconnaissance des populations musulmanes de la ville. De leur côté, les réfugiés ont également obtenu la jouissance gratuite de locaux où des activités religieuses et culturelles se déroulent régulièrement, mais cette dotation se fit, cette fois, dans l'indifférence générale.

Le statut plutôt protégé des réfugiés n'est toutefois pas immuable. Danièle Lochak(5), comme d'autres observateurs, remarque que leur image aujourd'hui n'est plus aussi bonne que par le passé, les pouvoirs publics adoptant une attitude de plus en plus méfiante vis-à-vis de dits "faux réfugiés" accusés d'abuser du "droit d'asile". Il s'agit là d'une attitude prolongeant celle de la fermeture des frontières à l'immigration, que renforcent aujourd'hui les Accords de Schengen. Comme on peut le constater, ce travail sur les mots nous ramène fréquemment à considérer simultanément les trajectoires des réfugiés et celles des autres types de migration. 
On sait que le terme "immigré" est devenu une catégorie dévalorisante qui comprend aussi bien des gens ayant migré que leurs enfants, pourtant nés en France, mais considérés comme marqués par l'itinéraire (assimilé à une véritable disgrâce) de leurs parents. Il est aujourd'hui souvent associé à l'idée de non-appartenance nationale, à la question de l'exclusion sociale ou à celle de la (non-)participation citoyenne. Cette notion d' "immigré" semble maintenir sinon à l'extérieur, du moins en marge de la société, des hommes et des femmes qui en sont pourtant partie prenante(6).

Ce mot par lequel on désigne, en France, ceux par qui le problème semble venir, est donc "piégé", lourd de représentations présentes et passées. Le terme "immigrant", par comparaison, est plus neutre, et traduit sans doute la vision plus positive que l'on a des phénomènes migratoires outre-Atlantique. D'ailleurs, si les populations d'Asie du Sud-Est arrivées en Amérique du Nord à la fin des années soixante-dix et au cours des années quatre-vingt ont été accueillies, comme en France, au titre de réfugiés, elles sont rapidement devenues, aux yeux des autorités administratives chargées de l'accueil et de la première installation, des immigrants comme les autres, avec les mêmes droits, notamment celui d'accéder à la citoyenneté quelques années (trois ou cinq ans) après leur arrivée.

\section{Perception des groupes minoritaires}

Le concept sociologique de minorité est particulièrement riche en matière de recherche et permet de compléter ce que nous venons de développer. Le terme est intéressant ici non pas dans son sens statistique mais par son caractère relationnel. Les sociologues parlent d'ailleurs de relations majoritaires-minoritaires(7), relations réciproques mais néanmoins asymétriques, ou encore de situation minoritaire, pour insister sur la prise en compte des différents partenaires de la relation, du contexte socio-historique et des rapports de pouvoir qui le structurent. Dans la perspective développée par divers travaux sociologiques sur la question(8), les collectivités constituées à la suite du refuge en France de milliers de personnes venant d'Asie du Sud-Est sont devenues des minorités ethniques, des groupes minoritaires contraints de se forger une place tant aux côtés d'autres minorités (avec lesquelles les relations sont aussi à construire) qu'à l'égard du groupe majoritaire.

Qu'est-ce qui caractérise la situation minoritaire ? Essentiellement deux choses :

- Comme les mineurs par l'âge, les minoritaires sont souvent frappés d'incapacités, ils sont infantilisés, comme dans certaines situations de travail par exemple, mais aussi dans les relations avec les travailleurs sociaux ou encore avec les représentants d'administrations. Dans le meilleur des cas, ils sont l'objet de sollicitude, comme s'ils avaient besoin d'être "sous tutelle".

- Les membres des groupes minoritaires sont souvent perçus comme "particuliers", affublés d'un ou plusieurs particularismes face à un groupe majoritaire qui, lui, incarne la généralité, la norme. Ils sont donc perçus comme différents, d'une différence souvent négative, mais pas toujours. On entend souvent mentionner, en même temps ou successivement, des différences positivement connotées, depuis les compétences musicales ou sportives des "Blacks", des "Beurs" ou des boxeurs thaïs, jusqu'aux compétences laborieuses des femmes "asiatiques" (minutieuses, précises, courageuses à la tâche) en passant par les critères esthétiques qui, dans certaines circonstances où, par exemple, la relation inégale se double d'une domination homme-femme clairement établie, l'exotisme devient particulièrement séduisant.

Différence positive ou négative, cette pensée de la différence est étroitement liée à l'idée que les groupes existent en soi, comme des entités fermées sur elles-mêmes, pourvues d'une vitalité intrinsèque, alors que ces groupes n'existent et ne se pérennisent qu'en vertu de rapports constitutifs avec les autres groupes. C'est donc l'étude de ces rapports, de leur nature et de leurs transformations, qui seule peut nous aider à saisir dans toute sa complexité la place de ces collectivités au sein de la société mais aussi le vécu, les trajectoires des individus et de leur famille au sein de ces groupes plus ou moins fortement constitués. 


\section{Groupe dominant et dynamiques identitaires}

Si ces rapports entre majoritaires et minoritaires nous permettent de comprendre la manière dont les groupes se forment et se maintiennent, ils influencent aussi directement les processus d'identification et la cristallisation de l'identité ethnique autour de marques (valeurs, pratiques ou critère distinctif au fort potentiel à la fois différenciateur et identificatoire) dont le choix reflète directement la nature de ces rapports. Inventions de "traditions" vestimentaires ou culinaires, pratiques ordinaires érigées en rites ou en symboles culturels chez les Lao par exemple: aujourd'hui, un certain nombre de comportements, de pratiques et de valeurs peuvent ainsi faire l'objet d'un surinvestissement parce qu'ils apparaissent compatibles tant avec les nouvelles valeurs qui pénètrent progressivement les foyers lao qu'avec la perception française de l'exotisme asiatique. Ces pratiques prennent une telle importance qu'elles deviennent de véritables traditions propres à forger une personnalité collective positive, épurée de tout ce qui pourrait devenir, en France, un élément stigmatisant et serait alors susceptible de nourrir une identité négative. Si l'on a pu ainsi mesurer le poids direct ou indirect du groupe dominant sur les dynamiques identitaires des réfugiés, qu'en est-il de leurs enfants ? Vivent-ils au quotidien des discriminations telles que l'identification à leurs amis de parents français leur apparaît comme interdite ou bien, au contraire, s'acheminent-ils plus tranquillement vers des compromis culturels dont les ressorts identitaires sont plutôt riches et variés ? L'étude reste à faire comme elle a été menée, sur divers terrains, auprès des enfants d' "immigrés".

Angélina Étiemble(9) en donne un exemple local à travers les discours recueillis auprès de jeunes femmes dont les parents marocains vinrent naguère s'installer à Rennes. Grâce à un travail ethnosociologique de longue haleine, elle montre combien les processus d'identification sont profondément structurés par l'inégalité des rapports entre majoritaires et minoritaires. Leurs discours sont en effet marqués par un double refus, celui d'adhérer aux valeurs patriarcales défendues par les parents (à propos du mariage notamment) et celui, apparemment contradictoire, de trahir leur appartenance à un groupe profondément "minorisé", à leurs yeux, au sein de la société française. Ces jeunes femmes vont donc développer toute une rhétorique où, s'interdisant de partager les valeurs et normes de la société française, elles en viennent à affirmer une identité à la fois féminine et marocaine. Le devenir des membres des minorités issues des migrations d'Asie du Sud-Est est encore, de ce point de vue, incertain. À contre-courant de l'illusion ahistorique qu'entretiennent les groupes sur leur propre existence et sur leur ethnicité, il faudra continuer d'être attentif au temps et aux processus qu'il engendre, soit bien embrasser, dans chaque situation, les forces du passé, les forces du présent, mais aussi celles de l'avenir.

1)- Olivier Brachet, "L'impossible organigramme de l'asile en France. Le développement de l'asile au noir", Revue européenne des migrations internationales, vol. XIII, 1997.

2)- Hélène Bertheleu, Organisation collective et ethnicité. Minorité lao à Rennes, Grenoble et Montréal, thèse de doctorat, université de Haute-Bretagne, Rennes-II, 1994, 466 p.; "Cohésion sociale, ethnicité et hiérarchies : fêtes et rituels lao en France", Revue européenne des migrations internationales, vol. XVI, $\mathrm{n}^{\circ}$ 2, 2000, pp. 153-170.

3)- Chantal Robineau, "Réfugiés d'Asie du Sud-Est : les groupes d'accueil en Ille-et-Vilaine", Pluriel, ${ }^{\circ} 28,1981$, p. 61. Cet accueil a compris différentes facettes : le logement, l'emploi, l'aide financière, les vêtements, l'aide aux démarches administratives, le tout devant aider la famille à progressivement devenir autonome.

4)- Ida Simon-Barouh, "Eux et Nous. Rennes et les étrangers", Les Cahiers de Rennes, n 2, 1987.

5)- Danièle Lochak, Étranger, de quel droit?, Puf, Paris, 1985.

6)- Voir René Gallissot, "Immigré/immigrant", Pluriel recherches. Vocabulaire historique et critique des relations interethniques, cahier $\mathrm{n}^{\circ} 1,1993$, pp. 65-68. 
7)- Colette Guillaumin, L'idéologie raciste. Genèse et langage actuel, Mouton, Paris-La Haye, 1972.

8)- Lire Pierre-Jean Simon, "Minorité”, Pluriel recherches. Vocabulaire historique et critique des relations interethniques, $\mathrm{n}^{\circ} 3,1995$, pp. 50-61.

9)- Angélina Étiemble, "Discours féminin sur le mariage : rhétorique et identité "marocaine"”, in Hélène Bertheleu (dir.), Identifications ethniques. Rapports de pouvoir, Compromis, Territoire. Actes des Journées Universitaires d'Automne 1999, Paris, L'Harmattan, 2001, p. 171-192. 